Bewegungskultur als elementarer Baustein sozialer und individueller Gesundheit: Was können wir von den Aboriginal People Australiens lernen?

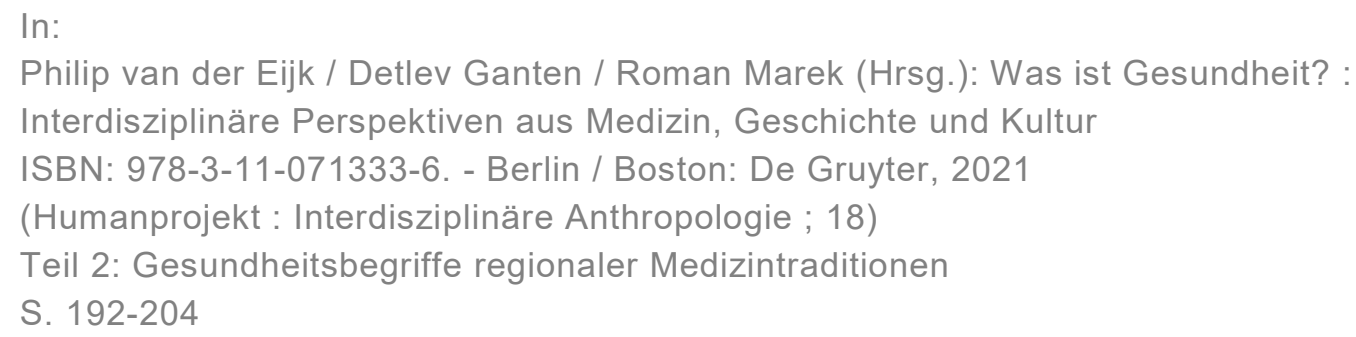




\title{
Bewegungskultur als elementarer Baustein sozialer und individueller Gesundheit: Was können wir von den Aboriginal People Australiens lernen?
}

\begin{abstract}
A Movement Culture as an Elementary Component of Social and Individual Health: What Can We Learn from the Aboriginal People of Australia? The Aboriginal People's traditional movement culture is part of the oldest health concept known to man. What can we still save and take on for our society today?
\end{abstract}

Die indigenen Völker Australiens hatten keinen Begriff für „krank“. Gesundheit bezieht sich bei ihnen nicht auf das Wohlbefinden des Einzelnen, sondern auf die ganze Familie und den Stamm. Körper, Geist und die sozialen Beziehungen unterliegen ihrer Vorstellung nach einer steten Dynamik zwischen Leben und Tod (Wrogemann 2000a, S. 11). Doch ein langer Prozess gewaltsamer Umbrüche hat heute die älteste Bewegungs- und Medizinkultur der Menschheit fast restlos ausgelöscht. Die australischen Ureinwohner selbst verfügten über keine Schrift. Wissen und Werte wurden mündlich oder in Form von Kunst, Gesängen und ihrer traditionellen Bewegungskultur von Generation zu Generation weitergegeben. Roth (Roth 1897, S. 152-166) dokumentiert seine teilnehmenden Beobachtungen zu Disease, Accident, Death, Canibalism in seinen ethnologischen Studien von Aboriginals in North-West-Central Queensland und gibt dabei auch Hinweise auf die Bewegungskultur. Diese Erfahrungen sind leider heute in vergleichbarer Weise nicht mehr möglich und größtenteils nur noch aus der historischen Literatur zu interpretieren. Wenige Australier befassen sich mit diesem Thema. Eine Ausnahme ist der Sportpädagoge Ken Edwards, der sich mit seiner Veröffentlichung Choopadoo: Games from the Dreamtime (Edwards 1999), der Thematik annahm und gemeinsam mit seinem Sohn Tim Edwards (Edwards \& Edwards 2011) ein ganz eigenes pädagogisches Sportprogramm für die direkte praktische Umsetzung z.B. in Kindertagesstätten und Schulen entwickelte (Edwards 2009, 2012). Darüber hinaus sind es leider nur wenige Initiativen, die einzelne Aspekte indigener Gesundheitskonzepte (wenn auch modifiziert) bewahren helfen. So starteten Wissenschaftler 1986 ein Projekt zum Sammeln der Heilmethoden austra-

Ohle Wrogemann, Bundesarbeitsgemeinschaft für Haltungs- und Bewegungsförderung e.V. (BAG), Wiesbaden

Ә OpenAccess. (C) 2021 Ohle Wrogemann, publiziert von De Gruyter. (cc) BY-NC-SA Dieses Werk ist lizenziert unter einer Creative Commons Namensnennung - Nicht kommerziell - Weitergabe unter gleichen Bedingungen 4.0 International Lizenz. https://doi.org/10.1515/9783110713336-015 
lischer Ureinwohner. Bereits damals wurde zurecht befürchtet, dass das jahrtausendealte Wissen australischer Heilpraktiken in den nächsten zehn Jahren verlorengehen könnte. Die Wissenschaftler reisten in entlegene Gebiete des Northern Territory und befragten die Stammesältesten $\mathrm{zu}$ den bis dato nur mündlich überlieferten Heilmethoden mit Hilfe pflanzlicher, tierischer und mineralischer Produkte (Blackburn 1988). Heutzutage erfährt die Busch-Medizin traditioneller Heiler, z. B. der Ngangkari, zunehmend Wertschätzung. So werden die Ngangkari durch eigene staatliche Gesetze, die ihre Qualifizierung und Anerkennung regeln, geschützt und gefördert. Sie führen eigenständig Behandlungen durch und stellen ihre kulturspezifischen Kompetenzen Aboriginal-Patienten zur Verfügung. Die Versorgung findet dabei in Einrichtungen des modernen australischen Gesundheitssystems statt. Die Anangu Ngangkari Tjutaku Aboriginal Corporation (ANTAC) in South Australia stellt diesen Gesundheitsservice dabei auch Australiern europäischer Abstammung zur Verfügung (ANTAC 2020). Andere traditionelle Heiler tragen in ihrer Bezeichnung meist einen regionalen Bezug, etwa „maban man“ und „jamandji doctor“. Sie sind mit Schamanen anderer Naturvölker vergleichbar, da sie auch spirituelles Wissen anwenden und soziale Konflikte regeln (Burnum 1988).

Doch weshalb spielt gerade die Bewegungskultur eine so wichtige Rolle im Leben des Einzelnen und für die Gesellschaft im Allgemeinen? Basierend auf der primären Sozialisation, in der sich - meist in der Familie - Persönlichkeitsmerkmale wie Sprache, Denken, Wissen und Emotionen ausbilden, lernt der Mensch in der sekundären Sozialisation durch die ihn umgebende Gesellschaft ein neues Rollenverhalten, mit dem er sich in die Kultur seiner Heimat einfügt. Salter (Salter 1967) machte in der einschlägigen Literatur 94 hierfür relevante Spiele und Freizeitaktivitäten ausfindig und konnte sie fünf Hauptkategorien zuordnen: ökonomische, politische und häusliche Aktivitäten, zeremonielle Identifikation und soziale Interaktion. Auf spielerische Weise werden somit Rollenverhalten und kulturelle Werte eingeübt, die für das Funktionieren einer Gesellschaft von essentieller Wichtigkeit sind. Dementsprechend sind auch die traditionellen Bewegungsspiele australischer Ureinwohner elementare und integrative, identitätsstiftende Bestandteile ihrer jeweiligen Stammeskultur (Wrogemann 1989). Heutzutage spielen soziale Einrichtungen wie Kindertagesstätten, Schulen und Sportvereine eine bedeutende Rolle in der sekundären Sozialisation von Heranwachsenden. Sport ist das Schulfach, in dem auch in Australien bildungsbenachteiligte Gesellschaftsgruppen, wie z.B. Aboriginal-Kinder, oft erfolgreicher sind als in kognitiven Unterrichtsfächern. Gerade deshalb hat der Sportunterricht einen besonderen pädagogischen Wert und er bietet eine ausgezeichnete Chance, bildungsbenachteiligte Kinder zu erreichen und zu ermutigen. 
Die Bewegungskultur der Aboriginals als Teil ihres traditionellen Gesundheitskonzepts hat es bis auf wenige Referenzen nicht in die moderne australische Gesellschaft geschafft. Gründe dafür sind nicht nur die größere Attraktion des Sports europäischer Prägung und eine Politik der Akkulturation, sondern auch die fehlende Möglichkeit, auf eine Dokumentation zurückgreifen zu können, da der traditionellen Kultur eine eigene Schrift fehlt. Ein Großteil des Wissens über die traditionelle Bewegungskultur stammt von Aufzeichnungen aus europäischer Perspektive und von Sportwissenschaftlern, die nur vergleichsweise wenige Zeitzeugen befragen konnten. Heute gibt es jedoch zunehmend erstarkende politische Interessenvertretungen, die versuchen, sich gegenüber einer akkulturierenden Bildungspolitik durchzusetzen. Um die Verwobenheit der Bewegungskultur mit den gesamtgesellschaftlichen Entwicklungen besser nachvollziehen zu können, soll hier zunächst eine kurze Zusammenfassung der Lebensumstände australischer Ureinwohner erfolgen.

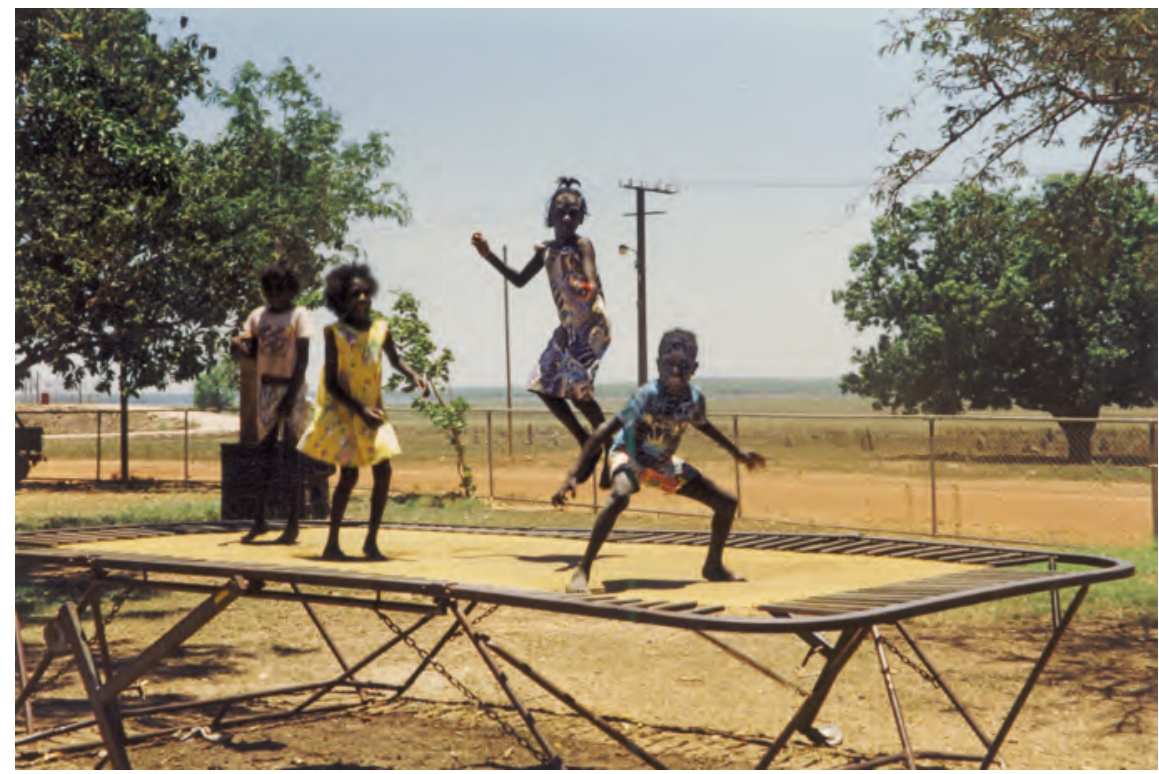

Abb. 1: Keine Chance für traditionelle Spiele: Mit Leichtigkeit und Spaß springen die Kinder auf ihrem Schulhof in Oenpelli im Arnhemland (Northern Territory) auf dem Trampolin, einem unglaublich attraktiven Gerät der dominierenden Bewegungskultur „Sport“. Foto: Ohle Wrogemann 


\section{Von der Kolonialisierung zum neuen Selbstverständnis}

Der Begriff „Aborigines“ gilt in Australien aufgrund seiner kolonialen Vergangenheit heute als diskriminierend. Aboriginal People oder Black Fellas, so bezeichnen sie sich selbst, zählten je nach Quelle bis zu 700 Stämme, die auch als Clans bezeichnet werden. Es existierten rund 200 bis 300 Sprachen mit etlichen Dialekten. Immer mehr der indigenen Sprachen sterben aus, weniger als 20 werden noch gelehrt. Mit jeder verloren gegangenen Sprache verschwindet auch das darin gespeicherte Wissen. Gleichzeitig entstehen auch neue Sprachen, wie Kriol im Norden von Queensland (Meakins 2014). Entsprechend ihrer Sprachzugehörigkeit und Heimatregion nennen sich die ersten Australier zum Beispiel Koori in Victoria und New South Wales, Murri in Queensland und im Nordwesten von New South Wales sowie Yolngu im North Arnhem Land im Northern Territory. Mit ihren Eigenbezeichnungen drücken sie ihre Identifikation mit ihrer Ethnie und vor allem ihren Stolz darauf aus. Der australische Staat bezeichnet die Ureinwohner des Kontinents zusammenfassend als „Aboriginals“. In diesem Begriff sind oft auch die Ureinwohner der Torres Strait Islands im Norden des Kontinents zwischen Queensland und Papua-Neuguinea miteingeschlossen.

Nach der britischen Eroberung des heute als Australien bezeichneten Kontinents ab 1788 wurden die indigenen Völker nach unserem heutigen Verständnis enteignet, von ihrem Land vertrieben und bis über die Mitte des 20. Jahrhunderts hinaus ermordet. Dennoch scheuen sich viele Autoren nach wie vor, den Begriff „Genozid“ zu verwenden, da sie, ohne es zu leugnen, u.a. das moderne Australien nicht mit diesen Gräueltaten in Verbindung bringen wollen (Tatz 1999). Im 19. Jahrhundert wurden die Stämme in sogenannten Reservaten konzentriert und vermischt. In den ihnen zugewiesenen Gebieten waren sie weit entfernt und abgeschnitten von ihren angestammten Lebensräumen, ihrer Ernährung und sogar von Wasserquellen, so dass sie abhängig von Nahrungsmittellieferungen der Regierung wurden. In den Jahren von etwa 1905 bis 1970 wurden, gemäß behördlicher Anweisungen des Australischen Staates, der Bundesstaaten und der Kirchen, zehntausende Aboriginal-Kinder ihren Eltern gewaltsam entzogen, um sie in Heimen, Missionen und bei weißen Familien umzuerziehen. Heute kämpfen Menschenrechtsorganisationen wie z.B. die Gesellschaft für bedrohte Völker (GfbV) für die Anliegen australischer Ureinwohner. Derartige NGOs und vor allem auch die Indigenen selbst haben in den vergangenen Jahrzehnten für eine größere Anerkennung ihrer Kultur gesorgt. Der inzwischen enorm gestiegene Bevölkerungsanteil an Ureinwohnern in Australien ist dafür ein ausschlaggebendes Indiz: Aboriginal People haben aufgrund der politisch deutlich verbesserten Bedingun- 
gen ein neues Selbstbewusstsein erlangt, werden nicht mehr verleugnet und müssen sich auch selbst nicht mehr aus Scham verleugnen. Ihre Kultur ist Teil der Identität aller Australier geworden.

Aktuell leben insgesamt rund 25 Millionen Menschen in Australien. Die Bevölkerungszahl der Ureinwohner bei Ankunft der Eroberer wurde je nach Quelle auf zwischen 300.000 und 1 Million geschätzt. Im Jahr 1901 wurden sie auf rund 67.000 geschätzt und 1930 nur noch auf 50.000. Die Volkszählung 1991 ermittelte 265.000 Aboriginals und 2006 waren es 464.000. Heute leben rund 800.000 Aboriginal People und Torres Strait Islander in Australien - die Prognose ist steigend. Als Aboriginal wird eine Person definiert, die von Aboriginals oder Torres Strait Islanders abstammt, sich als Aboriginal identifiziert und von einer Gemeinschaft, wie einem Clan, als Aboriginal anerkannt wird. Damit hat ein anerkannter Aboriginal alle Rechte, als solcher von den Regierungsstellen behandelt zu werden. Berndt und Berndt (Berndt \& Berndt 1986) unterscheiden „modern-traditional Aboriginals“, die ein modifiziert traditionelles Leben zurückgezogen auf ihrem Land führen, und „modern-urban Aboriginals“, die sich einem europäischen Lebensstil angepasst haben. Für die Einschätzung der Lebendigkeit der traditionellen Kultur ist diese Unterscheidung bedeutend: Im Jahr 1960 schätzte man die Zahl derer, die ein modifiziert-traditionelles Leben führen, auf rund 7.500 .

\section{Wenn die Lebensumstände nicht mehr zur Biologie passen}

Die Aboriginal People leben (je nach Quelle) seit ca. 40.000 bis 60.000 Jahren in Australien. Doch es sind gerade die letzten 250 Jahre, die maßgeblich ihre traditionellen Lebensweisen und ihren Gesundheitszustand zerstört haben. Immer mehr Aboriginals wurden sesshaft. Mit dem Einsatz von Gewehren zur Jagd wurden die natürlichen Nahrungsquellen im Busch bis zum Mangel reduziert. Die Jäger und Sammler mussten in den oftmals unwirtlichen Reservaten schließlich immer weiter hinausziehen, um traditionelle Nahrungsmittel $\mathrm{zu}$ finden. Diese schafften dann oftmals nicht mehr den Weg zurück zu den Kindern und Älteren, die sich zunehmend mit für sie unnatürlichen Lebensmitteln wie Mehl, Zucker und Konservennahrung ernähren mussten. Als Folge der unausgewogenen, kohlenhydratreichen und eiweißarmen Ernährung sowie des Bewegungsmangels stieg die Zahl der Fälle von Diabetes, Adipositas, Hypertonie und weiteren degenerativen metabolischen und kardiovaskulären Erkrankungen dramatisch an. Neville White (White 1985) spricht von biologischen Konsequenzen der Akkultu- 
ration, wobei Männer verhaltensbedingt eher darunter leiden als Frauen und Kinder. Denn während Männer sich an den Wochenenden in die Städte begeben, um beliebten australischen Freizeitbeschäftigungen (z. B. Australian Rules Football) nachzugehen und dort $\mathrm{zu}$ essen, verpflegen sich die Frauen und Kinder zuhause mit hochwertiger Nahrung, die sie im Busch sammeln. Der Gesundheitsstatus der Aboriginals entspricht dem der ärmsten Länder der Welt. Wie Grattan (Grattan 2020) berichtet, sind die Regierungsprogramme, die die Lücke zur Mehrheitsgesellschaft schließen sollen, mehrheitlich gescheitert. Die Maßnahmen hatten zum Ziel, u.a. die Kindersterblichkeit und die Lebenserwartung der Aboriginals, die 8 Jahre niedriger ist als die der Australier europäischer Abstammung, anzupassen. Macniven et al. (Macniven et al. 2019) analysieren Sportprogramme mit gesundheitlichen Zielsetzungen. Sie zeigen, dass die Programme zur Verbesserung des Bildungsniveaus, zum Aufbau von psychosozialen Ressourcen und zu kriminalpräventiven Maßnahmen Defizite aufweisen und nicht sehr erfolgreich sind.

Folglich assoziiert die australische Bevölkerung ihre Ureinwohner im Allgemeinen mit Krankheit, Drogen und Gewalt und stellt sie somit an den Rand der Gesellschaft. Armut, Ausgrenzung, Rassismen, interkulturelle Konflikte u.a. m. belasten die Clans, die Familien und den Einzelnen schwer. Die sogenannten Reports der australischen Regierung zur gesundheitlichen Lage der Aboriginals, die regelmäßig vom Australian Institute of Health and Welfare veröffentlicht werden, geben ein detaillierteres Bild über den Prozess. Zahlreiche Quellen dokumentieren die etwa doppelt so hohen Suizid-Raten von erwachsenen Aboriginals und Aboriginal-Kindern. Interventionsprogramme stehen hier vor großen Herausforderungen. Oft legen Medien in der Berichterstattung ihren Fokus nicht auf die wesentlichen Hintergründe, sondern auf Tragik und Schuldfrage. Manipulierte pseudo-wissenschaftliche Studien leisten hierzu noch ihren Beitrag (Wrogemann 2000a, S. 14). Gleichzeitig wird die Selbstverwaltung der Aboriginals in ein schlechtes Licht gerückt. Abwertung erzeugt weitere Abwertung - so besteht auf beiden Seiten eine gruppenbezogene Menschenfeindlichkeit, die verschiedenste Formen von Diskriminierung zur Folge hat. Zum Schutz der indigenen Völker vor dem Coronavirus verkündet der australischen Premierminister Reiseeinschränkungen in ihre Gebiete (AFP 2020) und bekräftigt in einer Pressekonferenz nach einer Kabinettssitzung zur Gesundheit der indigenen Australier, diese partnerschaftlich mit ihnen gemeinsam verbessern zu wollen (The Prime Minister of Australia 2020). 


\section{Die traditionelle Bewegungskultur der australischen Ureinwohner}

In der relativ kurzen Zeit seit der gewaltsamen Umsiedlung aus ihrer Heimat veränderte sich die traditionelle Bewegungskultur der australischen Ureinwohner radikal. Die Jäger und Sammler unter ihnen orientierten sich eher hin zu einer europäisch geprägten Bewegungskultur, die von den Traditionen und Werten unserer Leistungsgesellschaft beeinflusst ist. So dient der europäisch geprägte Sport als Medium zur Akkulturierung der Ureinwohnergesellschaft und unterstützt damit die Politik von Staat und Kirchen (Wrogemann 2000b, S. 304-311). Die traditionelle Bewegungskultur der Aboriginals war hingegen weniger an Wettbewerb und herausragenden Leistungen interessiert, sondern sie entwickelte sich im Sinne der Gemeinschaft: Fitness und Fair Play spielten eine große Rolle. Die Bedeutung einer gesundheitsorientierten Bewegungsförderung der Kinder war den australischen Ureinwohnern bewusst. Die Gemeinschaft forderte darüber hinaus zur Beteiligung aller auf. Zahlreiche Spiele wurden mit viel Spaß von Kindern und Erwachsenen gemeinsam gespielt (Edwards 1999, S. 2-3).

Die Bewegungskultur eines Aboriginal-Stammes steht prinzipiell in enger Beziehung zu der umgebenden Natur, d.h. seiner geographischen Lage und den dort herrschenden klimatischen Bedingungen. Aus Rohstoffen der Natur entwickelten sie ihre materiellen Kulturgüter, die sich auch in der Bewegungskultur wiederfinden, u.a., um den Umgang damit zu üben. Die Materialien der Bewegungskultur, etwa Waffen, erscheinen auf den ersten Blick einfach (Wrogemann 1989, S. 38 - 57). Sie sind jedoch Multifunktionsgeräte, die mit großem Geschick bis zur technischen Perfektion gefertigt werden und die nicht nur bei der Jagd auf Wild zum Einsatz kommen. Zu religiösen Zwecken, wie bei Ritualen der Initiation, sind sie oft mit Farben dekoriert. So werden sie heute auch als Souvenirs an Touristen verkauft. Als zweckdienliche Werkzeuge sind sie von schlichter Erscheinung. Der Speer ist beispielsweise in unterschiedlichsten Ausführungen überall in Australien zu finden. Er dient zum Jagen, Fischen, zur Selbstverteidigung, als Spielzeug und auch als Utensil zur Bestrafung. Speere werden mit unterschiedlichen Techniken geworfen. Sie werden bspw. vor dem Abwurf in Schwingung versetzt oder mit dem Ende in eine Speerschleuder - ein verlängerter Wurfarm, der häufig mit dem Wort Woomera bezeichnet wird - eingelegt, um damit einen kräftigeren Abwurf zu ermöglichen.

Auch die Woomera ist wiederum ein vielseitiges Werkzeug, das nicht überall in Australien zu finden ist. Es wird zum Feueranzünden durch Sägebewegungen auf weichem Holz benutzt, um Früchte von Bäumen zu holen, als Schale, Messer, Spaten, Schaufel und anderes mehr. Speerspiele von Jungen wurden auf dem 
ganzen Kontinent beobachtet. Als Ziele dienten dabei über den Boden gerollte Scheiben aus Rinde oder Büschel von Pflanzen, die an windigen Tagen davonwehen. Speerspiele wurden von Jungen und Männern als Mannschaftsspiele gespielt, die über Stunden andauerten. In Western Australia wurde das Speerspiel Malu genannt, was Känguru bedeutet. Dabei wurde eine ellipsenförmige Scheibe über den Boden gerollt, diese schien dann wie ein Känguru zu springen. Die Intention ist eindeutig - spielerisch werden motorische Fertigkeiten im Speerwerfen für die Jagd nach Nahrung erworben, doch auch für den Kampf bei ernsthaften Konflikten oder freundschaftlichen Turnieren. Gould (Gould 1970) beschreibt verschiedene Taktiken der Kriegsführung. Mit dem Speerwurf versuchte der Krieger, seinen Feind lediglich am Oberschenkel zu verletzen und so kampfunfähig zu machen. Gelegentlich kam es dabei aber doch auch zu Todesopfern. Ein Ngatatjara-Aboriginal in Western Australia berichtet von einer Taktik, bei der die Speere zwischen zwei Zehen eingeklemmt durch das Gras gezogen wurden, bis sich der Gegner auf eine gewisse Distanz genähert hatte.

Der Bumerang, bei uns als Sport- und Spielgerät bekannt, ist mehr als das Wurfinstrument, das in der Sprache der Dharuk-Aboriginals, die in der Gegend um Sydney ansässig waren, so bezeichnet wurde. Das Gerät wurde auch unter anderen Bezeichnungen, wie Kylie oder Kailee, geführt und hat verschiedenste Funktionen: Es dient zum Abschlagen von Gras, zum Ebnen von Flächen für Lager, Spiele und Zeremonien, zum Feuer entzünden sowie als Kochlöffel, Feuerschürer, Schaufel und Musikinstrument. Im Kampf wird der Bumerang in erster Linie als Schlag- und nicht als Wurfwaffe eingesetzt. Er ist in Australien, auch aufgrund der limitierten Handelswege zwischen den Stämmen, wenig verbreitet und variiert in Form und Größe. Im Westen von Queensland und in einigen Regionen von South Australia waren Bumerangs in einer Länge von bis zu zwei Metern in Gebrauch. Genaues Beobachten ihrer Natur und Umwelt sowie handwerkliches und künstlerisches Geschick bewegte Aboriginals im Süden von Viktoria zum Konstruieren von wiederkehrenden Bumerangs. Sie beobachteten, wie die Blätter eines Eukalyptusbaumes heruntersegelten und sich dabei drehten. Wenn ein Blatt gerade nach vorne geworfen wurde, machte es eine Kurve und kam zurück (McGivern 1972, S. 113).

Ihr Wissen bezogen die australischen Ureinwohner aus dem genauen Analysieren der physikalischen Bewegungen ihrer Umwelt, des Verhaltens von Tieren und der Eigenschaften von Pflanzen. Sie nutzten außerdem Wind und Thermik, Wasser und Strömung sowie unzählige weitere physikalische Zusammenhänge. Mit Wasser und dem Ozean waren die Aboriginals schon seit zehntausenden von Jahren vertraut, als sie mit Kanus aus Südostasien den Kontinent erreichten. Die meisten Ureinwohner lebten in der Nähe von lebensnotwendigem Süßwasser. Natürlich dienten die Gewässer auch als Nahrungsquelle, so schwammen, 
tauchten und fuhren sie darauf zum Jagen, Fischen und Sammeln ihrer Lebensmittel. Salter (Salter 1967, S. 173) beschreibt, wie Aboriginals Wasserschildkröten im Meer jagen. Sie klammern sich am Panzer fest und tauchen mit der Schildkröte in die Tiefe, um sie zu töten. Dabei bleiben sie geschätzte ein bis zwei Minuten unter Wasser. Mit Intelligenz und motorischen Fertigkeiten entwickelten sie Verhaltensweisen und auch Spiele für Kinder, die sensibilisieren und vor Gefahren schützen sollen. Im und um das Wasser sind das z. B. Brandung, Strömungen, Strudel, Tiefen, Krokodile und Haie. Spiele (Roth 1984 [1902], S. 10), wie das Fangenspiel „Haifisch“, werden von kleinen Kindern gespielt. Ein Mädchen oder ein Junge imitiert dabei die Bewegung eines Haies und versucht, die Spielkameraden im Wasser zu fangen. Das „Krokodilspiel“ ist am Princess Charlotte Bay beliebt. Hier tauchen die Kinder eine Strecke und lassen dabei Luftblasen, wie es ein Krokodil tut, nach oben steigen. Bei anderen Wasserspielen wird nach Gegenständen getaucht, von Felsen und Bäumen hineingesprungen, an glitschigen Böschungen hineingerutscht und auf kleinen Kanus aus Rinde gepaddelt. Die Nulakun-Aboriginals im Northern Territory hielten mit ihren Kindern einen formellen Schwimmunterricht ab, um ihnen die Nahrungssuche unter Wasser beizubringen (Moncrieff 1966, S. 9). Nach einem Badeunfall wurden alte Frauen beobachtet, wie sie jemanden mit einer Mund-zu-Nase-Methode wiederbelebten. Sie saugten an der Nase, und pressten und rollten den Körper, um das Wasser herauszubekommen (Salter 1967, S. 173). Die Mythologie der Aboriginals, unter den Stichworten Dreamtime oder Dreaming bekannt, nimmt u.a. auch Bezug auf Gefahren im Wasser. Die Aboriginals sprechen Tabus durch religiöse Regeln aus, wie das Verbot einen Wasserfall nicht als Spielplatz zu nutzen. So sind bspw. Wasserlöcher heilig und nicht zu betreten, weil das Wasser als Trinkwasser genutzt wird und durch das Plantschen der Kinder verunreinigt werden könnte (Wrogemann 1989, S. 62).

Ein weiteres prägendes Element sind größere Kulturfeste der australischen Ureinwohner, die sogenannten Corroborees. Die Anlässe können verschieden sein: religiöse Initiationen, Hochzeiten und Trauerfeiern. Sie können für Frauen, Männer und Kinder getrennt sein, können gemeinsam mit anderen Stämmen der Pflege ihrer Nachbarschaft dienen, sind teils geheim oder offen für alle, wenn die Unterhaltung im Vordergrund steht. Bei Corroborees mit Musik, Tanz und Pantomime, die über mehrere Tage gehen können, nehmen viele Hundert Personen teil, wobei es keine strikte Trennung zwischen Akteuren und Zuschauern gibt. Da die Aboriginals über keine Schrift verfügen, dienen die Events außerdem dem Informationsaustausch. Lieder und Tänze vermitteln Regeln und Verhaltensweisen. Es werden Artikel gehandelt, Spiele und Bewegungsformen vermittelt. Auch heute noch werden Corroborees gefeiert: z. B. Sports Carnivals wie in Barunga und Aboriginal Olympics in Yuendumu. Auf dem Programm stehen dabei neben Musik 
und Tanz versportete Wettkämpfe in traditionellen Bewegungsformen: Speerzielwurf auf eine Känguruattrappe, Demonstrationen und Meisterschaften im Feuermachen sowie Australian Rules Football und Volleyball. Doch über allem steht das soziale Miteinander. Die Besucher kommen von weither und benötigen oft Tage für die Anreise. In der traditionellen Ureinwohnergesellschaft hatte das Gewinnen und Verlieren anders als bei unserem Sport oft keine Bedeutung. Die aus unserer Sicht Besseren zeigten eher demütiges Verhalten und beschenkten beispielsweise die schlechteren Mitspieler mit einer schönen Feder. In unserem Sinne von schneller - höher - weiter wurde nicht gezählt oder gemessen, es sind Kriterien unserer europäisch geprägten Bewegungskultur. Dennoch zeigt sich auch bei den australischen Ureinwohnern ein gewisser Anspruch, Ehrgeiz oder Leistungsgedanke, etwa bei manchen traditionellen Bewegungsformen oder auch in der technischen Perfektion bei der Herstellung von Waffen (Wrogemann 1989, S. $63-67,78-86,90)$.

\section{Resümee: Was können wir von der Bewegungskultur der australischen Ureinwohner lernen?}

Erfolgreiche Mikroprojekte wie die von Edwards (Edwards 1999) zeigen, dass der Sportunterricht in den Schulen der australischen Ureinwohner durchaus traditionelle Inhalte integrieren kann. Der Sportpädagoge Peter Markey (Markey 1994) verdeutlicht andererseits in seiner kritischen Auseinandersetzung die grundsätzliche Problematik um die Medien Bewegung und Sport bei der gesellschaftlichen Integration von australischen Ureinwohnern, die auf Defizite des Selbstbewusstseins und schließlich gruppenbezogene Menschenfeindlichkeit zurückgehen. Dennoch hat der Sport in Verbindung mit der kulturellen Identität positive Effekte und schafft Persönlichkeiten mit Vorbildcharakter. Die traditionelle Bewegungskultur war so reichhaltig, dass sie trotz veränderter Lebensbedingung weiter hätte bestehen können. Nach dem Land Right Act leben viele Aboriginals wieder in der Heimat ihrer Vorfahren. Kinder, die am Wasser leben, könnten die alten Spiele zur Sensibilisierung von Gefahren spielen, sich aber auch neue Spiele ausdenken. Bei diesem Aufwachsen in der Natur würden sie sich auch gesünder entwickeln.

Auch in Deutschland zeigt sich eine vergleichbare Problematik: Der Mensch leidet unter der Umgebung, die er selbst geschaffen hat. Der Körper passt nicht mehr zur Umwelt. Bereits unsere Kinder weisen erhebliche und zunehmende Defizite in Bezug auf eine gesunde Entwicklung auf (Robert Koch-Institut 2020): 
So bemängelt das Gesundheitssystem bei Kindern eine hohe Anzahl degenerativer Erkrankungen, damit sind auch die zunehmenden Störungen des Sozialverhaltens und der Psyche gemeint, die u.a. auf den Mangel an Bewegungserfahrungen zurückzuführen sind. Die Politik hat den zwar wichtigen Digitalpakt für Schulen durchgesetzt, gemäß ihrer Fürsorgepflicht für die Kindergesundheit hätte aber zunächst ein Bewegungspakt beschlossen werden müssen. In RheinlandPfalz soll dieser für die Kindertagesstätten mit einem Konzept zur Bewegungskindertagesstätte (Bewegungskindertagesstätte Rheinland-Pfalz e.V. 2020) folgen. Der organisierte Sport, der richtigerweise ein lebenslanges Sporttreiben propagiert, ist zwar das größte bürgerschaftliche Netzwerk in Deutschland, kann aber eine umfassende Prävention quantitativ unmöglich leisten. Er ist vornehmlich durch die Mittelschicht vertreten und propagiert darüber hinaus einen ebenso teuren wie imageträchtigen Bereich: den Spitzensport. Viele gesellschaftliche Gruppen sind aus unterschiedlichsten Gründen vom Sport ausgegrenzt und haben daher lebenslang die gesundheitlichen Folgen $\mathrm{zu}$ tragen. Eine allgemeine Teilhabe an einer Bewegungskultur im Sinne von Gesundheitsgerechtigkeit muss also unabdingbarer Teil eines sozialen Gesundheitskonzepts sein.

Bewegung ist elementar und entscheidend für einen ganzheitlichen Entwicklungsprozess zum gesunden Menschen und zum Erhalt seiner Gesundheit. Die Konzepte dazu liegen längst vor. So gibt Balster (Balster 2003) praktische Hilfen zur Förderung der Wahrnehmung und Bewegungsentwicklung, die leicht an die Bedürfnisse von z. B. Senioren angepasst werden können. Das Konzept von Waldkindergärten und Waldpädagogik ist aktueller denn je, da hier auf eine Nachhaltigkeit im Umgang mit einer für die Menschheit überlebenswichtigen Ressource hingearbeitet wird. Die traditionelle Kultur der Aboriginals liefert unzählige Erkenntnisse und relativiert zugleich die Forderung nach kostenintensiven Lernräumen, die von einer pädagogischen Architektur künstlich entwickelt wurden: Das Setting Natur bietet einen Bewegungsraum für alle. Dennoch: Dass es hierzu bei uns eines Handlungsplanes über alle Generationen hinweg bedarf, steht außer Frage. Es gilt sich nun den Herausforderungen zu stellen und Vertrauen aufzubauen. Denn unsere Gesellschaft differenziert: in der Bildung, im Beruf und auch im Sport. Gleichzeitig wird sie durch Globalisierung, Migration und Individualisierung immer heterogener. Mit der ökonomischen Ungleichheit geht oft eine kulturelle Ungleichheit und Ungleichbehandlung einher. Die negativen Erscheinungen, wie das Ausspielen von Personen und Gruppen gegeneinander durch Ausgrenzung, Vorurteile und Schuldzuweisungen ist eine altbekannte Masche bestimmter politischer Gruppierungen, die gesundheitlichen Folgen davon haben wir alle zu tragen. Der Blick in die Welt und auf andere Bewegungs- und Medizintraditionen, wenn auch retrospektiv, zeigt, dass es auch anders geht. Die nähere Betrachtung der inklusiven Bewegungskulturen austra- 
lischer Ureinwohner zeigt Lösungsmöglichkeiten für einen Weg hin zum Ideal „Gesundheit für alle“. Es ist höchst bedauerlich, dass die indigenen Australier selbst ihre heutzutage wieder höchst geschätzten Werte nie selbst kennengelernt haben. Sie hatten nie eine Schrift, haben die meisten Sprachen verloren und ihre Bewegungskultur vergessen. Dennoch haben sie es geschafft, sich als Gemeinschaft wiederzufinden und sich eine hoffentlich gesündere Zukunft zu sichern.

\section{Literatur}

AFP (2020): „Coronavirus: Australia Restricts Travel to Indigenous Areas to Protect Aborigines“. The Straits Times vom 20.3.2020. https://www.straitstimes.com/asia/australianz/ australia-restricts-travel-to-indigenous-areas-over-coronavirus-fears.

ANTAC (2020): „Ngangkari Services“. https://www.antac.org.au/ngangkai-services/ngangkariservices, besucht am 12.05.2020.

Balster, Klaus (2003): Kinder mit mangelnden Bewegungserfahrungen. Teil 2. Praktische Hilfen zur Förderung der Wahrnehmung und Bewegungsentwicklung (3. Aufl.). Duisburg: Sportjugend im Landessportbund Nordrhein-Westfalen e. V.

Berndt, Catherine Helen/Berndt, Ronald Murray (1986): Aborigines in Australian Society. Melbourne: Pitman.

Bewegungskindertagesstätte Rheinland-Pfalz e.V. (2020): „Bewegungskita RLP“. https://www. bewegungskita-rlp.de/, besucht am 15.06.2020

Blackburn, R. (1988): „40,000 Years of Healing in Print“. The Australian vom 9.9.1988.

Burnum, Burnum (1988): Burnum Burnum's Aboriginal Australia. A Traveller's Guide (David Stewart, Hrsg.). North Ryde: Angus \& Robertson.

Edwards, Ken (1999): Choopadoo: Games from the Dreamtime. Brisbane: Queensland University of Technology Publications.

Edwards, Ken (2009): „Traditional Games of a Timeless Land: Play Cultures in Aboriginal and Torres Strait Islander Communities“. In: Australian Aboriginal Studies 2, S. 32- 43.

Edwards, Ken (2012): A Typology of the Traditional Games of Australian Aboriginal and Torres Strait Islander Peoples. Esk: Ram Skulls Press, https://eprints.usq.edu.au/24916/12/ Edwards_typology_PV.pdf, besucht am 14.06.2020.

Edwards, Ken/Edwards, Tim (2011): A Bibliography of the Traditional Games of Torres Strait Islander Peoples. Toowoomba: Faculty of Education, University of Southern Queensland, https://eprints.usq.edu.au/24915/7/Edwards_Edwards_Games2011_PV.pdf, besucht am 14.06.2020.

Gould, Richard A. (1970): „Spears and Spear-Throwers of the Western Desert Aborigines of Australia“. American Museum Novitates 2403 vom 18.2.1970, S. 1-42.

Grattan, Michelle (2020): „,Closing the Gap“ Report Shows Disappointing Results“. City News. https://citynews.com.au/2020/closing-the-gap-report-shows-disappointing-results/, besucht am 12.4.2020.

Macniven, R./Canuto, K./Wilson, R. et al. (2019): „The Impact of Physical Activity and Sport on Social Outcomes among Aboriginal and Torres Strait Islander People: A Systematic Scoping Review“. In: / Sci Med Sport 22(11), S. 1232-1242. 
Markey, Peter Edward (1994): „Sport, Physical Education and Academic Success: Playing on the Same Team for Aboriginal Students?“ [M. A. Thesis, Northern Territory University]. Darwin.

McGivern, Muriel Evelyn (1972): Aborignal of the Dandenong Mountain. Melbourne: Melbourne Printers.

Meakins, Felicity (2014): „Australiens Aborigines erfinden ganz neue Sprachen“. Die Welt. https://www.welt.de/kultur/article134054781/Australiens-Aborigines-erfinden-ganz-neueSprachen.html, besucht am 10.04.2020.

Moncrieff, John (1966): „Physical Games and Amusements of the Australian Aboriginal“. In: The Australian Journal of Physical Education 36, S. 5-11.

Robert Koch-Institut (2020): KIGGS - Studie zur Gesundheit von Kindern und Jugendlichen in Deutschland. Berlin: Robert Koch-Institut, https://www.kiggs-studie.de/deutsch/home. html, besucht am 14.06.2020.

Roth, Walter Edmund (1897): Ethnological Studies Among the North-West-Central Queensland Aborigines. Brisbane: Government Printer.

Roth, Walter Edmund (1984 [1902]): „Games, Sports and Amusements“. In: Ken F. MacIntyre (Hrsg.): The Queensland Aborigines, Bd. 2. Carlisle: Hesparian Press, S. 10-11.

Salter, Michael. A. (1967): „Games and Pastimes of the Australian Aborigines“ [M. A.-Thesis, University of Alberta]. Edmonton.

Tatz, Colin (1999): „Genocide in Australia“. In: Journal of Genocide Research 1(3), S. 315-352. The Prime Minister of Australia (2020): „Update on Coronavirus Measures. Media Statement 12. Jun 2020: Closing the Gap“. https://www.pm.gov.au/media/update-coronavirusmeasures-12june20, besucht am 14.06.2020.

White, Neville G. (1985): „Sex Differences in Australian Aboriginal Subsistence: Possible Implications for the Biology of Hunter-Gatherers“. In: J. Ghesquiere/Robert D. Martin/F. Newcombe (Hrsg.): Human Sexual Dimorphism. London: Taylor \& Francis, S. 323-361.

Wrogemann, Ohle (1989): „Die Bewegungskultur der australischen Ureinwohner (Aborgines)“ [Magisterarbeit, Justus-Liebig-Universität Gießen]. Gießen.

Wrogemann, Ohle (2000a): „Der Sport und die traditionelle Bewegungskultur der Aborigines“. [Inaugural-Dissertation, Justus-Liebig-Universität Gießen]. Gießen.

Wrogemann, Ohle (2000b): „Sport as a Tool to Erase the Traditional Culture of the Aborigines and Torres Strait Islanders“. In: Manfred Messing/Norbert Müller (Hrsg.): Blickpunkt Olympia: Entdeckungen, Erkenntnisse, Impulse. Kassel: AGON Sportverlag, S. 304-311. 